Departamento de Educación Médica, Facultad de Medicina, Universidad de Concepción, Chile.

asicóloga, Magíster en Ciencias de la Educación Mención Didáctica e Innovación Pedagógica.

${ }^{\text {b} M a t r o n a, ~ M a g i ́ s t e r ~ e n ~}$ Educación, mención Currículum.

'Bioquímico, Magíster en Educación Médica para las Ciencias de la Salud. dPsicólogo, Magíster en Psicología Educacional

eEnfermera, Magíster en Enfermería.

${ }^{\text {f} M e ́ d i c o ~ C i r u j a n o, ~ M a g i ́ s t e r ~ e n ~}$ Educación Superior.

Ingeniero Informático, Magíster en Educación Médica para las Ciencias de la Salud.

hEnfermera, Magíster en Educación Médica para las Ciencias de la Salud. 'Psicóloga, Magíster en Psicología Educacional.

Trabajo financiado por proyecto FONDECYT No1140654.

Recibido el 10 de julio de 2015, aceptado el 9 de marzo de 2016.

Correspondencia a : Eduardo Fasce $\mathrm{H}$. Departamento de Educación Médica, Facultad de Medicina, Universidad de Concepción. Barrio Universitario $\mathrm{s} / \mathrm{n}$ Concepción Chile. efasce@udec.cl

\section{Aspectos motivacionales involucrados en el aprendizaje autodirigido en estudiantes de medicina. Un enfoque cualitativo}

\author{
EDUARDO FASCE H., JAVIERA ORTEGA B. ${ }^{\text {a }, ~ P I L A R ~ I B A ́ N ̃ E Z ~ G . ~}{ }^{\text {, }}$ \\ CAROLINA MÁRQUEZ U.c , CRISTHIAN PÉREZ V. ${ }^{\text {, }}$ \\ CAROLINA BUSTAMANTE D. ${ }^{\mathrm{e}}$, LILIANA ORTIZ M. ${ }^{\mathrm{f}}$, \\ OLGA MATUS B. ${ }^{\mathrm{g}}$, NANCY BASTÍAS V. ${ }^{\mathrm{h}}$, CAMILA ESPINOZA P. ${ }^{\mathrm{i}}$
}

\section{Motivation and self-directed learning among medical students}

Background: Motivation is an essential aspect in the training process of medical students. The association that motivation can have with learning self-regulation is of utmost importance for the design of curriculum, teaching methods and evaluation. Aim: To describe the motivational aspects of self-directed learning among medical students from a traditional Chilean University. Material and Methods: A qualitative, descriptive study based on grounded theory of Strauss and Corbin. Twenty $4^{\text {th }}$ and $5^{\text {th }}$ year medical students were selected using a maximum variation sampling technique. After obtaining an informed consent, semi-structured interviews and field notes were carried out. Data were analyzed to the level of open coding through Atlas-ti 7.5.2. Results: From the student point of view, personal motivational aspects are linked to the search for information, constant updating, the perception of the physician-patient relationship and interest in subject matters. From the scope of teachers, a main issue is related to their ability to motivate students to develop independent study skills. Conclusions: Personal motivational aspects facilitate the development of independent study skills, specifically in the search of information. The role of teachers is crucial in promoting these skills and the perception of medical students from their learning process.

(Rev Med Chile 2016; 144: 664-670)

Key words: Learning; Motivation; Students, Medical.
E 1 desarrollo de habilidades de aprendizaje autodirigido puede ser fundamental para el desarrollo profesional, entendiendo este constructo como la utilización de estrategias cognitivas, metacognitivas y motivacionales que utilizan los estudiantes para aprender continuamente ${ }^{1}$. Actualmente, el cambio constante que ocurre en el conocimiento científico requiere de la generación de prácticas educativas que estimulen habilidades de aprendizaje que sirvan a los estudiantes para analizar la información continuamente, para lo cual es difícil sustentar la educación basándose en la réplica de los modelos docentes pasados ${ }^{2}$, y por tanto, es esencial contar con docentes autodidactas en su disciplina ${ }^{2,3}$.

Existe evidencia sobre la relación que tiene el aprendizaje autodirigido con variables como el perfil sociodemográfico y académico ${ }^{4,5}$, los estilos y estrategias de aprendizaje ${ }^{6,7}$, metacognición ${ }^{8}$ y factores predicitivos de índole afectiva y académica, 
evidenciando que estudiantes autodirigidos presentan mayor dirección de su propio aprendizaje, confían más en sus capacidades para enfrentar la realidad, planifican de manera efectiva sus tiempos, además de involucrarse efectivamente en ellos ${ }^{9-11}$. Asimismo, se ha observado una serie de factores psicoeducativos que pueden influir en la disposición o interés por aprender, ya sean motivacionales, relacionales o afectivos ${ }^{12}$. Otros factores pudiesen afectar negativamente este proceso, por ejemplo, cuando los instrumentos de evaluación se vuelven rutinarios y monótonos, promoviendo la pasividad en el aprendizaje ${ }^{13}$.

De esta forma, el aspecto motivacional cobra una real importancia en los procesos de adaptación en situaciones cambiantes, sobre todo cuando se presentan discrepancias en el bienestar personal ${ }^{14}$. De acuerdo a Maslow, la motivación corresponde al "por qué" del comportamiento humano y se dirige a satisfacer necesidades básicas entre las cuales se incluye lo cognitivo y el aprendizaje $^{15}$. Pittman, Emery y Boggiano ${ }^{16}$ planteaban la diferencia entre la motivación intrínseca y la extrínseca, enfatizando que la primera se asocia a elementos como la novedad, la complejidad, el desafío y la oportunidad. Años después, surge la propuesta de Ryan y Deci ${ }^{17}$ con la teoría de la autodeterminación definiendo la motivación como un constructo caracterizado por energía, dirección, persistencia y equifinalidad, aspectos asociados a la activación y la intención que tienen los sujetos al interactuar con su entorno. Estos autores realizaron un enfoque de la motivación que diferencia los aspectos funcionales y experienciales de la auto-motivación y la regulación externa, los cuales tienen consecuencias específicas para el aprendizaje, el rendimiento, y la experiencia personal.

Considerando la importancia que tiene la motivación en el proceso de enseñanza-aprendizaje surge el interés de describir los aspectos motivacionales involucrados en las habilidades de estudio independiente, desde un enfoque cualitativo.

\section{Metodología}

Este estudio es una investigación cualitativa basada en la Teoría Fundamentada de Strauss y Corbin ${ }^{18}$, de tipo descriptiva y de alcance analítico relacional utilizando el método de comparación constante de estos ${ }^{18-20}$. Participaron 20 estudiantes de $4^{\circ}$ y $5^{\circ}$ año de la carrera de Medicina, escogidos mediante muestreo de máxima variación como arranque muestral ${ }^{19}$, identificando grupos heterogéneos y utilizando el muestreo teórico para alcanzar la saturación de datos ${ }^{18}$, previo proceso de consentimiento informado. Los participantes tienen una edad media de 23,3 ( $\mathrm{DE}=1,8)$, de los cuales $45 \%(n=9)$ son mujeres y $55 \%(n=11)$ son hombres.

Se utilizó la entrevista semi-estructurada, como técnica de recolección de dato ${ }^{20}$. El análisis de datos se realizó a partir del método de comparación constante de Strauss y Corbin ${ }^{18}$ en el nivel de codificación abierta, el cual permite identificar categorías a partir del contenido señalado por los sujetos participantes y relacionarlas. Los datos fueron procesados en el software Atlas-ti 7.5.2.

El estudio fue aprobado por los Comités de Ética de la Universidad ejecutora, y visado por el Comité Asesor de Bioética de CONICYT.

\section{Resultados}

A partir de los datos analizados, se obtuvieron un total 2.210 códigos en vivo, lo que permitió ser fiel al discurso inicial de los participantes y contribuyó a comprender las temáticas generales que se generaron a partir de las entrevistas semi-estructuradas. En el nivel de codificación abierta ${ }^{18}$ emerge la categoría "Aspectos motivacionales del aprendizaje autodirigido" en estudiantes de medicina, la que incluye dos subcategorías: "Aspectos personales de los estudiantes vinculados en la motivación intrínseca" y "Rol docente en la motivación extrínseca". Estas categorías se describen a continuación y son ilustradas con viñetas textuales extraídas de las entrevistas, las que se exhiben en las Tablas 1 y 2.

\section{Aspectos personales que motivan a los estudiantes de medicina al aprendizaje autodirigido}

Existen diversos aspectos personales que motivan a los estudiantes a desarrollar habilidades de estudio independiente. Uno de ellos tiene relación con la necesidad de buscar autónomamente fuentes bibliográficas como un recurso al momento de querer ahondar sobre un tema, resolver dudas o dominar mejor un contenido. Los alumnos recurren a fuentes diferentes a las asignadas cuando presentan dudas, buscando profundizar en alguna 
Tabla 1. Viñetas textuales extraídas de las entrevistas que ilustran las categorías: aspectos personales que motivan a los estudiantes de medicina al aprendizaje autodirigido

\begin{tabular}{|c|c|}
\hline Número & Viñeta textual \\
\hline Cita 1 & $\begin{array}{l}\text { "Por ejemplo si hay algo que nunca había escuchado o un fármaco que nunca había leído, como que trato de } \\
\text { buscar qué es lo que es y para qué se ocupa"[Entrevistado 15, 4 año. Mujer, } 21 \text { años] }\end{array}$ \\
\hline Cita 2 & $\begin{array}{l}\text { "[Mi visión de la medicina influye] en fijarme en algunos libros que son mucho más básicos para buscar esos } \\
\text { detalles. Buscar, por ejemplo, en algunos manuales de otras carreras que puedan estar más enfocados a la } \\
\text { práctica del paciente, o sea a veces buscar en manuales de enfermería que son cosas que yo no voy a des- } \\
\text { empeñar pero que a veces tienen indicaciones muy claras de los tratamientos y cosas así que, que sirven un } \\
\text { poco más para, para orientar al paciente en eso y el resto es estudiar un poco más las aplicaciones extras o los } \\
\text { posibles tratamientos extras que podría tener la misma patología" [Entrevistado } 7,5^{\circ} \text { año. Hombre, } 23 \text { años] }\end{array}$ \\
\hline Cita 3 & $\begin{array}{l}\text { Entrevistador: ¿Y qué te hizo buscar actualizaciones, qué buscabas con eso tú? } \\
\text { Entrevistado 18: Eh, no sé, como estar al día, si me preguntan los doctores. Igual es más para formación propia, } \\
\text { para no andar perdido en el hospital de repente cuando no conozco algún fármaco, o alguna patología, los } \\
\text { busco en esos medios. [ } 4^{\circ} \text { año. Hombre, } 22 \text { años] }\end{array}$ \\
\hline Cita 4 & $\begin{array}{l}\text { "Encuentro que es más interesante y hay más cosas que quizás nunca había escuchado o que me gustaría } \\
\text { entenderlas mejor, entonces sí sé quizás más o menos pero quiero saber qué es lo que es, bien, entonces lo } \\
\text { busco más" [Entrevistado } 15,4^{\circ} \text { año. Mujer, } 21 \text { años] }\end{array}$ \\
\hline
\end{tabular}

Tabla 2. Viñetas textuales extraídas de las entrevistas que ilustran la categorías: aspectos del rol del docente que motivan a los estudiantes de medicina al aprendizaje autodirigido

\begin{tabular}{|c|c|}
\hline Número & Viñeta textual \\
\hline Cita 1 & $\begin{array}{l}\text { "No sé, por ejemplo cuando el doctor te da un ambiente grato, es así como ya voy a estudiar porque realmente } \\
\text { como que él se merece mi atención" [Entrevistado } 4,5^{\circ} \text { año. Mujer, } 22 \text { años] }\end{array}$ \\
\hline Cita 2 & $\begin{array}{l}\text { "[Con ese tipo de docentes] llego a la casa al tiro con ganas de estudiar, aunque esté muy cansado llego con } \\
\text { ganas de estudiar, en cambio el otro docente es como...ya, llego cansado, 'ya, tengo certamen ¿en cuánto?, } \\
10 \text { días, ya hoy día no estudio, descanso'" [Entrevistado 6, 50 año. Hombre, } 23 \text { años] }\end{array}$ \\
\hline Cita 3 & $\begin{array}{l}\text { "Si, es que en cirugía igual había gente que anda motivada, y eso, la primera vez me tocó un docente que } \\
\text { era asistente, súper joven que era recién egresado. Él me invitó por primera vez a un pabellón, y me gustó y } \\
\text { empecé a ir, solo después (...) me quedaba en urgencia, porque me transmitieron esa motivación al principio, } \\
\text { como que gracias a esto, a esta persona que me motivó como que me perduró" [Entrevistado } 5,5^{\circ} \text { año. } \\
\text { Hombre, } 24 \text { años] }\end{array}$ \\
\hline Cita 4 & $\begin{array}{l}\text { "yo creo que siempre en un ambiente más ameno uno va a aprender más y mejor, eso es lo que pienso yo, ¿por } \\
\text { qué?, porque al final uno se interesa más, le toma como cariño al tema, investiga por su cuenta" [Entrevistado } \\
8,5^{\circ} \text { año. Hombre, } 23 \text { años] }\end{array}$ \\
\hline Cita 5 & $\begin{array}{l}\text { "Bueno cuando uno tiene clases con doctores que ya uno sabe que son secos y que saben mucho, mucho y } \\
\text { que ayudan mucho al paciente, obviamente uno no quiere desteñir frente a ellos, no quiere quedar como 'no } \\
\text { sé nada'... entonces dijimos 'ya, voy a buscar más cosas" [Entrevistado } 13,4^{\circ} \text { año. Mujer, } 23 \text { años] }\end{array}$ \\
\hline Cita 6 & $\begin{array}{l}\text { "hay un caso de un paciente en el mismo caso que dice el jefe que tenía que ser [la amputación] arriba de la } \\
\text { rodilla pero el doctor en pabellón dijo 'no ya, me la voy a jugar bajo la rodilla' y le cortó abajo a ver si estaba } \\
\text { muy malo y si estaba malo lo cortaba arriba, pero no estaba tan malo así que lo dejó y el paciente se quedó } \\
\text { con la rodilla. Entonces, esas cosas hacen que [uno piense] 'ya si no, si cirugía sirve no es tan terrible'" [Entre- } \\
\text { vistado } 13,4^{\circ} \text { año. Mujer, } 23 \text { años] }\end{array}$ \\
\hline Cita 7 & $\begin{array}{l}\text { "Sí, pediatría me gustaba más y gine como que nunca me gustó mucho. Entonces yo creo que por eso también } \\
\text { tenía que ver con un tema como de motivación, y como por gusto personal y también tiene que ver con el } \\
\text { docente, que hay docentes que son más motivadores que otros" [Entrevistado } 10,5^{\circ} \text { año. Mujer, } 30 \text { años] }\end{array}$ \\
\hline
\end{tabular}


determinada temática, o buscando prepararse para situaciones en que serán evaluados por los docentes (cita 1, Tabla 1).

Un segundo aspecto que motiva el desarrollo de habilidades de estudio independiente, es el modo en que los estudiantes perciben la relación médico-paciente. Es en esta relación cuando los estudiantes logran discernir qué elementos deben tener en cuenta para entender la práctica clínica, ya que al vincularse con el paciente pueden entender el contexto del trabajo del médico y visualizan al otro en esta relación diádica, a quien perciben como su responsabilidad. Esto los lleva a una búsqueda activa de información que les permita resolver las necesidades de esta interacción, por lo que, cuando cuentan contextos asignados de carácter muy técnico o conceptual, recurren a otros textos que se orienten más a la práctica clínica y al trato del paciente (cita 2, Tabla 1).

Un tercer aspecto motivacional que caracteriza a los estudiantes autónomos es la actualización constante con respecto a los avances en medicina. Algunos estudiantes mencionan que actualizarse es útil para afrontar de mejor forma la experiencia clínica en el hospital, lo que implica conocimiento práctico que sea útil en el ejercicio cotidiano de la medicina, pero también conocimiento que permita prepararse con éxito ante las posibles interrogaciones de los docentes (cita 3, Tabla 1).

Por último, se torna relevante la preferencia o interés que sienten los alumnos con la materia o ramo en sí, ya que esto parece guiar la búsqueda de fuentes adicionales. Así, el que les interese un tópico los llevará a buscar más información buscando una comprensión más cabal y profunda de éste, aunque esto no les reporte mejores calificaciones o reconocimientos de sus docentes. El siguiente entrevistado menciona los motivos por los cuales ahonda más en la asignatura de medicina interna, y no así en las demás (cita 4, Tabla 1).

En suma, estas motivaciones personales son atribuidas por algunos entrevistados a su motivación por aprender y a la vocación con la medicina. Este interés sería incluso más importante que la utilidad de la información frente a una evaluación o si dicha información ha sido enseñada o no durante las distintas instancias académicas. Pero en este contexto, la motivación principal y que impulsa a las otras suele ser el deseo y la responsabilidad de ayudar al paciente, lo que trasciende las exigencias académicas.

\section{Aspectos del rol del docente que motivan a los estudiantes de medicina al aprendizaje autodirigido}

Más allá de los elementos que permiten describir al docente que motiva, que implica establecer una relación con los alumnos cordial, respetuosa y agradable, en la que se sienten tratados como personas, los estudiantes también se refirieron al efecto que este tipo de docente tiene en su proceso de aprendizaje. En términos generales, y tal como lo indica su nombre, en este contexto, los alumnos mencionan que un docente motivador genera en ellos el interés por estar atento y mostrar una actitud positiva durante sus asignaturas (cita 1, Tabla 2).

Asimismo, el docente que motiva hace que los alumnos sientan ganas por estudiar. En este marco, mencionan que a diferencia de los demás docentes, cuando el docente motiva, sienten un interés genuino por aprender sobre los contenidos que enseñan. Por ello, destinan más tiempo para estudiar para esas asignaturas (cita 2, Tabla 2).

Así como mencionan sentirse más motivados para estudiar y preparar las clases de los docentes motivadores, los alumnos también reportan haberse sentido más interesados hacia el área que enseña dicho docente (cita 3, Tabla 2).

Este interés por el tema lleva a que los alumnos no sólo se sientan más motivados durante las clases, sino que busquen información adicional sobre el tópico, impulsándolos a buscar fuentes de manera autónoma, de modo de saber más sobre el tema (cita 4, Tabla 2).

$\mathrm{Al}$ momento de referirse a este interés por la búsqueda autónoma de fuentes, los alumnos declaran que con ello sienten la satisfacción de desempeñarse bien frente a esos docentes. De este modo, al ser estos docentes modelos a seguir en lo técnico y en lo valórico, los alumnos se exigen más a sí mismos, para mantener una imagen positiva frente a ellos (cita 5, Tabla 2).

La influencia del docente que motiva ha tenido importancia incluso en aquellas asignaturas en las cuales los alumnos no tenían interés o motivación inicialmente. De este modo, el docente que motiva es capaz de revertir la desmotivación de los alumnos (cita 6, Tabla 2).

Considerando lo anterior, el docente que motiva no sólo genera motivación en los alumnos por aprender, sino que también conlleva a que los estudiantes reporten mejores aprendizajes. 
Los mejores aprendizajes reportados por los estudiantes se desprenden, a su vez, de los efectos en la motivación que sienten los alumnos (cita 7, Tabla 2).

\section{Discusión}

Los resultados muestran que la autodirección en los aprendizajes está vinculada con los aspectos motivacionales que los estudiantes experimentan durante su proceso formativo ${ }^{17}$, involucrando tanto aspectos gatillados por características del estudiante como del docente.

De esta forma, se presenta evidencia que apoya la noción que el ambiente educativo y el interés personal del docente por enseñar y por lograr que los estudiantes aprendan favorecerían el desarrollo de habilidades de estudio independiente. Es así como, según la propuesta de Pittman, Emery y Boggiano $^{16}$, el docente que tiene las características antes mencionadas podría promover una motivación extrínseca en el proceso de aprendizaje de los estudiantes. Lo anterior se evidencia en como el estudio autónomo y el logro de mayores competencias por parte del estudiante son vistas como una estrategia para lograr una buena imagen ante el docente motivador, lo que a su vez permite mantener con éste una relación significativa que se caracteriza por ser grata para el estudiante. Para el logro de lo anterior, el docente debe generar instancias de aprendizaje donde exprese una actitud positiva hacia los estudiantes, manifieste un interés genuino por enseñar y aprender, diseñe un proceso de enseñanza planificado e intencionado, esté consciente del rol de modelaje que cumple en lo técnico y práctico-valórico, y por último, evalúe las necesidades de los estudiantes.

Sin embargo, este efecto externalizante de los docentes sobre la motivación podría ser sólo inicial, ya que el docente motivador logra transmitir el interés por su área disciplinar y por la actualización en medicina, aspectos que con el tiempo pueden volverse intrínsecamente motivadores ${ }^{16}$. Es más, entendiendo este proceso en el esquema de necesidades de Maslow, podemos considerar que los docentes motivadores, que generan relaciones respetuosas con el estudiante y muestran un interés genuino por enseñar, permitirían satisfacer las necesidades de seguridad (al no ser maltratados) y de afiliación (al sentirse apreciados) del estudiante, permitiéndole -a partir de esta satisfacción- proyectarse a necesidades más elevadas como las de reconocimiento y autorrealización ${ }^{15}$. Esto se vería respaldado en el carácter central que adquiere la idea identificada en este estudio de "mejorar como médico, para mejorar para el paciente" como motivador en el ejercicio clínico.

Por otro lado, el interés personal que el estudiante tiene sobre su aprendizaje constituye un elemento fundamental de la autonomía en el aprendizaje. Así, los aspectos personales mencionados por los estudiantes hacen referencia a elementos esenciales de la motivación intrínseca ${ }^{21}$. Para esto, es fundamental incentivar que los estudiantes puedan realizar una constante búsqueda de información, apoyar el proceso de análisis crítico de fuentes bibliográficas y la discusión de disenso y consenso del estado del arte.

Asimismo, el estudio permite considerar la importancia que tiene la motivación en el desarrollo de habilidades de estudio independiente a lo largo de la carrera de medicina, toda vez que el interés por una mayor competencia y un mejor desempeño para el paciente, movilizan a que el estudiante por sí sólo se comprometa en alcanzar una mayor comprensión de las temáticas médicas (principalmente clínicas), y que se involucre en la búsqueda de información adicional que le permitan alcanzarla. Es aquí donde vemos que el estudiante autónomo logra plantearse sus propias metas de aprendizaje, más allá de las disposiciones del programa que estudia, y define estrategias para alcanzarlas, lo que es parte troncal de la definición del aprendizaje autodirigido ${ }^{1,4,5,7,9}$.

Lo anterior, se relaciona con un estudio realizado por Lueddeke ${ }^{21}$, demostrando que los modelos educativos que aceptan la variabilidad del desarrollo de los estudiantes durante su proceso de formación, tienden a lograr efectivamente sus objetivos, demuestran satisfacción personal, mayor motivación, mayor autoconfianza y mejor proyección en el futuro profesional.

Esto muestra como los factores ambientales (como el rol del docente, antes mencionado) y los factores individuales, no afectan la motivación ni el aprendizaje autodirigido de manera aislada. En esta línea, Ten Cate, Kusurkar y Williams ${ }^{22}$, enfatizan que la motivación y la autonomía de los individuos se debe desarrollar en escenarios educativos adecuados que se benefician cuando los docentes favorecen la autonomía, para lo cual 
es fundamental que los académicos cumplan un rol regulador en el proceso de aprendizaje. Por otra parte, se ha comprobado que el rol de docentes motivadores, a través de la incorporación de modelos pedagógicos adecuados, favorecen el desarrollo de la autonomía en sus estudiantes ${ }^{23}$.

En un estudio previo, observamos que el compromiso académico fue una de las variables más importantes para el logro conductual, mayor que el afectivo 9 . Si bien existen aspectos motivacionales que facilitan el desarrollo de habilidades de estudio independiente, específicamente en la búsqueda de información, el rol del docente es fundamental en la promoción de ellas y en la percepción que tienen los estudiantes de su proceso formativo. Por lo tanto, es de importancia considerar que las propiedades y dimensiones de los aspectos motivacionales involucrados en el aprendizaje autodirigido orientan sobre qué elementos se deben considerar para promover instancias que propicien un aprendizaje más autónomo, en donde el equipo académico emerge como un punto central a trabajar.

A partir de estos resultados surge el interés de capacitar a los docentes con el propósito de optimizar sus estrategias pedagógicas orientadas a favorecer en sus estudiantes las habilidades de estudio independiente. Sin embargo, los resultados muestran que dicha capacitación, más que aspectos puramente disciplinares, debe apuntar a desarrollar en los académicos habilidades pedagógicas y relacionales, que les permitan gestionar un ambiente de aprendizaje cálido, grato y respetuoso, que de esta forma se vuelva motivador, y desde ahí pueda promover la autodirección de los estudiantes ${ }^{23}$.

\section{Referencias}

1. Knowles MS. Self-directed Learning: A guide forlearners and teachers. N. Y. Cambridge Book Company, 1975.

2. Fasce E. ¿ Son suficientes las competencias profesionales en la enseñanza de las ciencias de la salud? Rev de EducCienc Salud 2008; 5: 81.

3. Castellanos C, Marques P, Rodríguez M, Romero R. Caracterización de la preparación del docente en el análisis de un problema de salud. Revista Cubana de Educación Médica Superior 2003; 17: 14-24.

4. Deyo Z, Huynh D, Rochester C, Sturpe D, Kiser K. Readiness for Self-directed Learning and Academic Per- formance in an Ability Laboratory Course. AJPE 2011; 75: 1-7.

5. Fasce E, Ortega J, Pérez C, Márquez C, Parra P, Ortiz L, et al. Aprendizaje autodirigido en estudiantes de primer año de medicina de la Universidad de Concepción y su relación con el perfil sociodemográfico y académico. Rev Med Chile 2013; 141: 1117-25.

6. Borracci R, Guthman G, Rubio M, Arribalzaga E. Estilos de aprendizaje en estudiantes universitarios y médicos residentes. Educ Med 2008; 11 (4): 229-38.

7. Márquez C, Fasce E, Pérez C, Ortega J, Parra P, Ortiz L, et al. Aprendizaje autodirigido y su relación con estilos y estrategias de aprendizaje en estudiantes de medicina. Rev Med Chile 2014; 142: 1422-30.

8. Jiang Y, Kleitman S. Metacognition and motivation: Links between confidence, self-protection and self-enhacement. Learning and Individual Differences 2015; 37: 222-30.

9. Spormann C, Pérez C, Fasce E, Ortega J, Bastías N, Bustamante $\mathrm{C}$, et al. Predictores afectivos y académicos del aprendizaje autodirigido en estudiantes de medicina. Rev Med Chile 2015; 143: 374-82.

10. Pintich P, De Groot E. Motivational and self-regulated learning components of classroom academic performance. J Educ Psychol 1990; 82: 33-40.

11. Schunk DH, Zimmerman BJ. Motivation and self-regulated learning: Theory, research, and application. Routledge, New York, 2008.

12. Covarrubias P, Martínez C. Representaciones de estudiantes universitarios sobre el aprendizaje significativo y las condiciones que lo favorecen. Perfiles Educativos 2007; 29 (5): 49-71.

13. Herrera J. Los juegos: una alternativa creativa en la enseñanza de la Traumatología. Rev Educ Cienc Salud 2012; 9: 36-42.

14. Reeve J. Motivación y Emoción. McGraw-Hill: México, 2003.

15. Maslow AH. Motivation and personality. Harper and Row, New York, 1970.

16. Pittman T, Emery J, Boggiano A. Intrinsic and extrinsic motivational orientations: Reward induced changes in preference for complexity. Journal of Personality and Social Psychology 1982; 42: 789-97.

17. Ryan R, Deci E. Self-determination theory and the facilitation of intrinsic motivation, social development, and well-being. American Psychologist 2000; 55: 68-78.

18. Strauss A, Corbin J. Bases de la investigación cualitativa. Universidad de Antioquía. Medellín 2002.

19. Flick U. Introducción a la Investigación Cualitativa. Morara. Madrid 2004.

20. Vieytes R. Metodología de la investigación en organi- 
zaciones, mercado y sociedad. Editorial de las Ciencias. Buenos Aires 2005.

21. Lueddeke G. Transforming Medical Education for the 21st Century. Megatrends, priorities and change. Radcliffe Publishing Ltd. United Kingdom, 2012.

22. Ten Cate, T Kusurkar R, Williams G. How self-deter- mination theory can assist our understanding of the teaching and learning processes in medical education. Medical Teacher 2011; 33: 961-73.

23. Piskurich JF. Fostering self-directed learning in medical school: when curricular innovation is not enough. Int J Self-Directed Learning 2011; 8: 44-50. 